\title{
Interfacial Water Studies and their Relevance for the Energy Sector
}

\author{
Alberto Striolo \\ Department of Chemical Engineering \\ University College London \\ London, UK, WC1E 7JE
}

\begin{abstract}
Interfacial water is ubiquitous, and its investigation has attracted the interest of many for both fundamental and applied purposes. This perspective provides a few personal highlights concerning how molecular, and sometimes multi-scale investigations on the properties of interfacial water could be of practical relevance for the energy sector. The discussion focuses on the transport of electrolytes through narrow pores, and on the solubility and transport properties of confined hydrocarbons, with particular attention in attempting to describe how interfacial water can affect such properties, especially within narrow pores. Recent results on the self-assembly of surfactants on heterogeneous surfaces are also briefly discussed. Finally, a few possible directions for future research are suggested. It should be stressed that this is not a comprehensive review on the possible impact of interfacial water studies on the energy sector, but rather a brief commentary on some personal interests, with the intent of stimulating debate and future studies.
\end{abstract}




\section{Introduction}

Computational modeling and simulation have evolved enormously since the pioneering activities in the middle of the twentieth century, when the first models for Lennard-Jones fluids and water models were introduced [1-4]. With the everincreasing computing power, and with the development of algorithms solidly built on statistical mechanics principles, modern researchers can provide important insights useful for a number of practical applications. While it is always useful to synergistically combine computational and experimental investigations, it should be recognized that computational approaches are now more than ever attempted to shed light on phenomena that cannot be studied via direct experimentation (e.g., Brennan and coworkers [5] are developing algorithms to study energetic materials).

Among research areas that are natural fields for computational research and that attract enthusiasm from multiple disciplines are solar cells [6], batteries [7] and energy storage in general [8], catalysis [9], medicine and biology [10]. The progress made by computational scientists in these areas is significant, and certainly not many were expecting such achievements only a few years ago.

In some cases, modeling and simulations are so advanced that they can trigger experimental investigations. One example of practical applications in which computational insights led experimental verifications and the design of new devices is that of water desalination [11], in which case simulation results have pioneered the use of novel materials (e.g., first carbon nanotubes and then graphene sheets) in advanced structured membranes [12,13]. In perhaps less glamorous, but more ubiquitous applications, computational modeling and simulations have traditionally provided the engineering community with insights regarding composition, structure and dynamics of more and more complex fluid mixtures [14-16]. This type of information has been used to design both novel separation strategies and novel materials.

The goal of this brief outline is to discuss some applications in the general area of the energy sector in which modeling and simulations can provide useful insights, and to identify fundamental questions that I consider fascinating yet helpful for securing progress. As already mentioned, areas such as the design of solar cells, catalytic materials, energy storage devices, etc., have attracted a lot of attention. Rather than commenting on these applications, the focus here will be on topics that, while remaining both challenging and at the scientific cutting edge, find applications in more traditional forms of the energy sector, including the oil and gas and the nuclear industries. In my opinion, answering some of these questions could lead to long-term environmental and economical benefits. While recognizing that many groups contributed to this field, the discussion here is a personal perspective. As such, this contribution does not represent a comprehensive review, but rather a short overview whose intent is to raise interest on the subject matter. 


\section{Discussion}

\section{a. Fundamental Studies on Interfacial Water}

Many investigated structure and dynamics of water at the interface with a solid substrate using a variety of computational techniques. Several reviews have been published on this topic [17-20].The simulation results suggest that the morphology of the solid substrate, the density and distribution of preferential adsorption sites, and even molecular-level details such as the direction of the dipole moments at the interface affect, and sometimes determine the properties of interfacial water. From the point of view of the computational community, one has to question whether the predictions from simulations are reliable towards understanding experimental results. In some cases, it has been reported that several water models yield somewhat similar results concerning structure and dynamics of water at interfaces [21-23], but some widely used water models yield differing results, in particular with respect to the dynamics of interfacial water. It has also been reported that when advanced techniques such as ab initio density functional theory (DFT) calculations are employed, results such as the energy of adsorption of one water molecule on a widely studied substrate such as graphite are strongly dependent on the models implemented in the calculations [24].

More encouraging is this scenario when molecular simulation results are compared, semi-quantitatively, to experimental data. In this case some promising comparisons have indeed been reported. For example, Mamontov et al. [25] combined experimental quasielastic neutron scattering data and molecular dynamics (MD) simulations to probe the dynamics of water on rutile. They found that the temperature dependence of the relaxation time for hydration water is strongly dependent on the amount of water present on the interface. This observation was taught to be associated with the ability of water molecules to jump between different hydration layers; these jumps are not possible when the hydration level is too low. Achtyl et al. [26] combined an interfacial potential-dependent version of second harmonic generation experiments with ab initio DFT calculations and reactive MD simulations to demonstrate that aqueous protons are able to transfer across single layer graphene, and that the transfer occurs through rare yet naturally occurring atomic defects. Argyris et al. [27] showed that simulation results can be helpful in reconstructing the three-dimensional layering of water at an interface based on experimental AFM data. In particular, it was shown that the probing AFM tip influences the interfacial water at small tip-surface separations [28]. These, and other promising results suggest that the simulation community could contribute to better understanding the role of interfacial water on applications of interest to the energy sector. To become really useful for practical applications, these types of investigations should consider solid substrates that are representative of sub-surface formations, which of course is problematic both for experiments and for simulations. However, it should be recognized that the investigations (both computational and experimental) conducted on perfect atomically smooth samples might not be representative of realistic conditions. For the oil and gas industry three types of reservoirs are of particular importance: the more traditional sandstones and carbonates, and organicrich shale rocks, which have attracted world-wide interest when the conjunction of 
technology advancements in different fields (i.e., horizontal drilling and hydraulic fracturing) allowed the USA to become the world's largest producer of oil and to reinvigorate its domestic chemicals industry [29]. For the nuclear industry it is of extreme importance to understand clays and how they interact with aqueous systems, because it is often proposed to use these materials as barriers to prevent the dispersion of nuclear waste from geological repositories. In the next section it is discussed how it might be possible to use the results from molecular simulations to provide useful insights for the energy sector.

\section{b. Possible Connections to the Energy Industry}

Regarding the use of clays as barriers for storing nuclear waste, Marry and Rotenberg recently summarized possible strategies that could be implemented to study, from the molecular to the macroscopic points of view, clays and the transport of various compounds through them [30]. Marry and Rotenberg highlighted the importance of capturing molecular details to describe the overarching properties of interesting materials such as clays, and they also discussed the state of the art regarding the multi-scale modeling of both the mechanical properties of clays, and the correlation between structure of clays, presence of water, and transport of electrolytes through clay substrates. The studies conducted to unveil the influence of the solid structure on the fluid properties at the clay-liquid interface [31,32], as well as attempts to improve our understanding concerning the electric double layer [33,34], are of particular interest here .

The types of studies just summarized tend to focus on one clay surface at contact with a thin film of aqueous solution, or, more often, one slit-shaped pore confined by two clay surfaces filled with aqueous solution of various electrolytes. This configuration can provide useful information, but the results it can provide might be strongly dependent on the system composition and the identification of experimental conditions representative of those sampled in the simulations might be problematic. Makaremi et al. [35], for example, recently investigated the swelling of montmorillonite and beidellite clays in the presence of water and $\mathrm{CO}_{2}$. They found that when only $\mathrm{CO}_{2}$ is present, clay swelling is thermodynamically unfavorable, result which points to the importance of hydration water on determining the properties of clays. For these calculations Makaremi et al. implemented the Gibbs ensemble Monte Carlo method [36], which allowed them to simultaneously calculate phase multicomponent equilibrium and swelling. An alternative approach is the grand canonical Monte Carlo method, which also allows to unambiguously identify the thermodynamic conditions of interest for equilibrium studies [37]. However, the increased availability of computing power has recently allowed the simultaneous simulation of bulk and confined systems within a single simulation box using equilibrium MD algorithms [38-40], although in this approach it might be problematic to allow swelling of the confining material. By conducting the MD simulations within the canonical ensemble (constant number of particles, constant volume and constant temperature) it is possible to identify the bulk conditions at equilibrium with a confined system [41]. In the case of clays, this approach has been for example implemented by Jardat et al. [42], who considered montmorillonite clay materials and provided a systematic investigation on the effect of pore size and salt concentration 
on the equilibrium distribution of ions between the slit-shaped pores and the bulk region. These approaches are becoming very attractive because they are relatively simple to use and they can provide pictorial representations of the phenomena under investigation, which is useful when attempting to bridge computational and experimental communities. MD approaches such as those just described could also be used to understand the resistances encountered by the ions as they transfer from the bulk regions to inside the pores [43]. The pathways followed by the ions tend to be those that encounter the minimal free energy barriers, and as such they depend on steric effects (e.g., the structure and dynamical properties of water molecules near the pore entrance) and on the presence of chemical groups on the pore entrance. To correctly describe these chemical groups in clays, one has to consider the chemical reactivity of the pore edges, for which purposes it is appropriate to implement DFT calculations [44-47]. Much remains to be done to better understand edges, defects, and the rate of dissolution of minerals. In particular, to enable the wide applicability of molecular simulations for practical applications in the energy sector, it will be important to develop atomistic force fields that can appropriately describe the edges. Zeitler et al. [48] developed parameters for describing the bending motion of $\mathrm{Mg}-\mathrm{O}-\mathrm{H}$ groups at the edges of brucite. The parameterization is compatible with the CLAY-FF force field [49], widely used to simulate systems containing clays, and the methodology could be applied to develop appropriate methods to describe the edges of other clays.

Regarding the transport of aqueous electrolytes through narrow slit-shaped pores, Argyris et al. [50] observed a strong correlation between the preferential position of an ion in the direction perpendicular to the pore surface and the ion mobility. These MD simulations were conducted for slit-shaped pores carved out of cristobalite, and although the results were highly dependent on the degree of protonation of the surface [51], overall they suggest that $\mathrm{Cs}^{+}$ions can dislocate much faster than $\mathrm{Na}^{+}$ ones through slit-shaped pores of narrow width because while the $\mathrm{Na}^{+}$ions tend to accumulate near the solid surface, where water molecules are almost immobile within the times accessible using MD simulations, while $\mathrm{Cs}^{+}$ions preferentially remain near the pore center, where water molecules can diffuse. These results are summarized in Figure 1, where both the density distribution of $\mathrm{NaCl}$ and $\mathrm{CsCl}$ electrolytes in the direction perpendicular to the pore surface, and the estimated selfdiffusion coefficients are reported as a function of the degree of protonation of the $\mathrm{SiO}_{2}$ cristobalite surfaces. It should be pointed out that $\mathrm{Cs}^{+}$ions have been found to accumulate near clay surfaces by other MD simulations [43], hence it would be of general interest to quantify the molecular reasons responsible for the different behavior of aqueous $\mathrm{Cs}^{+}$ions adsorbed within slit-shaped pores carved out of various solid materials. These types of studies could be of interest for the management of nuclear waste because $\mathrm{Cs}^{134}$ is one of the radionuclides present in nuclear waste, hence it is of interest to both separate it from other electrolytes, and prevent its dispersion in the environment. 

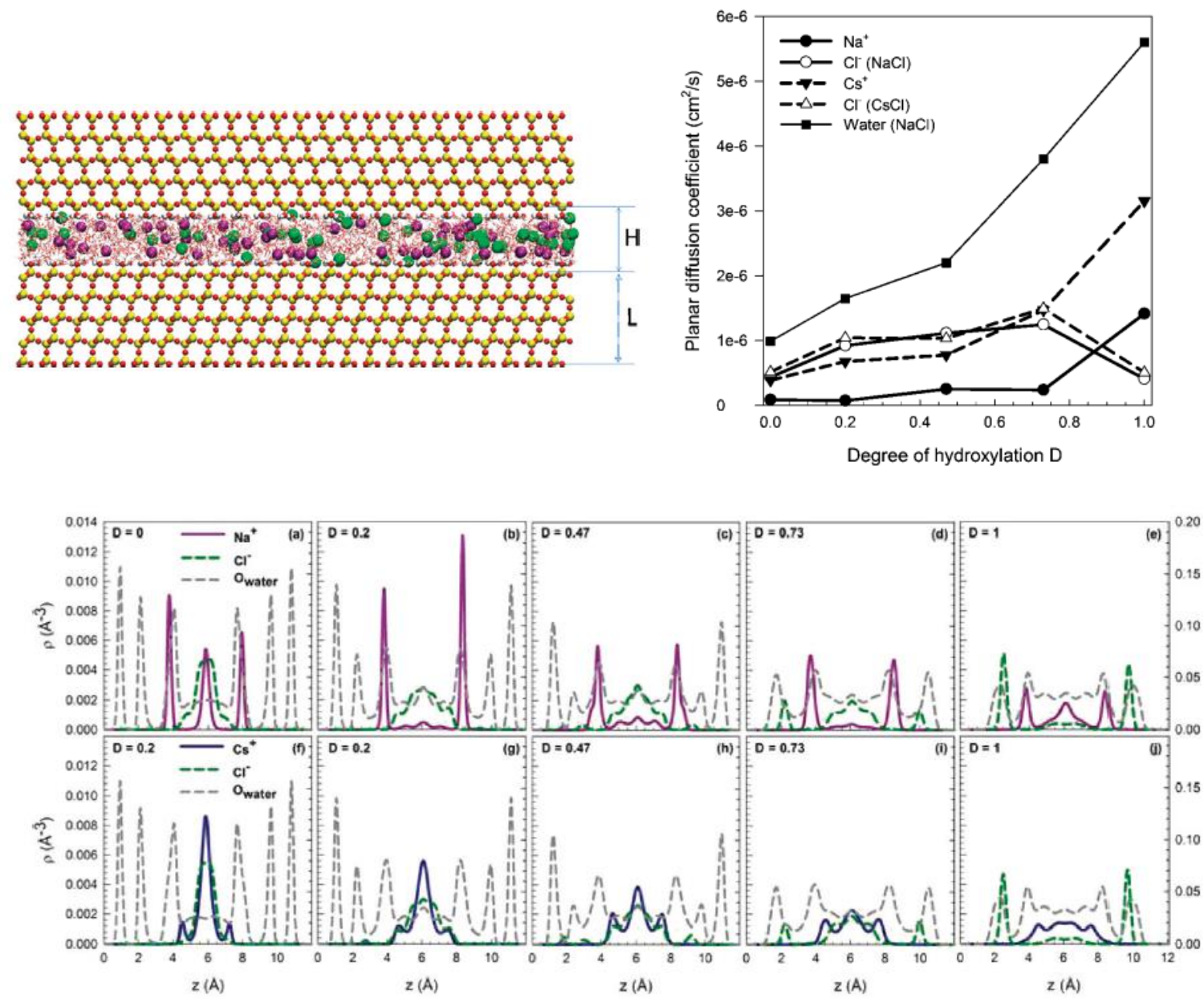

Figure 1. Top left: Simulation snapshot representing aqueous $\mathrm{NaCl}$ solution confined within a slit-shaped pore carved out of cristobalite. Top right: Planar self-diffusion coefficients calculated for $\mathrm{Na}^{+}, \mathrm{Cs}^{+}$and $\mathrm{Cl}$ - ions confined within the slit-shaped pore of width $\mathrm{H}=1.208$ $\mathrm{nm}$ as a function of the degree of protonation D. Bottom: density distributions in the direction perpendicular to the pore surface for $\mathrm{NaCl}$ and $\mathrm{CsCl}$ as a function of the degree of protonation of the pore surfaces. The density distributions for the $\mathrm{O}$ atoms of water molecules are also reported in this panel. Reproduced from Ref. [52].

The research just discussed focused on the effect of hydration water on determining the fate of electrolytes through narrow pores found in sub-surface formations or in barrier materials designed for preventing the ions from dispersing throughout the environment. It is also possible that hydration water might affect the transport and accumulation of hydrocarbons through sub-surface formations. This possibility needs to be explored because aqueous fluids are often used to stimulate sub-surface formations such as shale deposits to enhance the permeability of fluids, and also to recover hydrocarbons from partially depleted traditional reservoirs. To assess the likelihood of hydration water to facilitate or prevent hydrocarbon transport, one first- 
approximation approach could be to consider bulk-like properties to estimate the behavior of fluids within porous matrices. In this case, it is widely known that hydrocarbons are poorly soluble in water. However, one question that has always been of fundamental interest is how confinement affects the thermodynamic properties, and how the properties of confined fluids differ compared to those known in the bulk. For example, Makaremi et al. [35] calculated the solubility of $\mathrm{CO}_{2}$ in water confined within narrow clay pores using molecular simulations, and they found that, for pore swollen by water, the $\mathrm{CO}_{2}$ solubility under confinement was much larger than that in bulk water at both 25 and 125 bar at $348.15 \mathrm{~K}$. These results led to the suggestion that 'smectite minerals are good candidates for $\mathrm{CO}_{2}$ storage' [35]. As another example, Phan et al. [52] reported that, according to equilibrium MD calculations, methane might be much more soluble in water when the latter is confined in narrow, 1-nm wide pores than when it is at bulk conditions, at equal temperature and pressure. The results, reproduced in Figure 2, suggest that the confining material has a strong influence on methane solubility [53]. In fact, when slitshaped pores of $1 \mathrm{~nm}$ in width are considered, Phan et al. predict that the solubility is much larger when water is confined in silica pores than when it is confined in pores carved out of materials such as alumina and a model MgO substrate. Phan et al. [53] discussed the molecular reasons suspected to be responsible for these differences, including the density fluctuations triggered by the confining material and the network of hydrogen bonds formed among water molecules at the interface with the solid substrates. It is perhaps important to point out the possibility that these results are a consequence of the models implemented to describe water, water-methane, and water-rock interactions. As, according to Phan et al. [53], the structure of confined water is crucial for explaining the differences in methane solubility observed computationally, and since the structure of confined water is a strong function of the properties of the confining material, and it does not seem to depend to strongly on the models implemented to describe water,[22] one has to wonder how the uncertainties related to the description of the solid substrate and those connected with the description of water-solid interactions can affect the computational results. Given the present uncertainties, experimental validation is required to validate the molecular modeling predictions. Note that the simulation results by Makaremi et al. [35] seem to be in qualitative agreement with experimental observations [54]. While there is no doubt that a better fundamental understanding of aqueous systems under confinement, and the ability of describing quantitatively the solubility of hydrocarbons in confined water might have a strong effect in the environmentally conscious deployment of shale gas, because of the wide use of water in fracturing fluids, and of the possible natural presence of water within the sub-surface formations, as well as on designing reliable strategies for the long-term storage of $\mathrm{CO}_{2}$ in sub-surface formations, to be of practical relevance studies such as those of Phan et al. should be conducted for pores carved out of clays (one of the materials largely present in shale formations), should consider explicitly the barriers encountered by the gas molecules as they enter the pores, and should also address the transport of the hydrocarbons. 


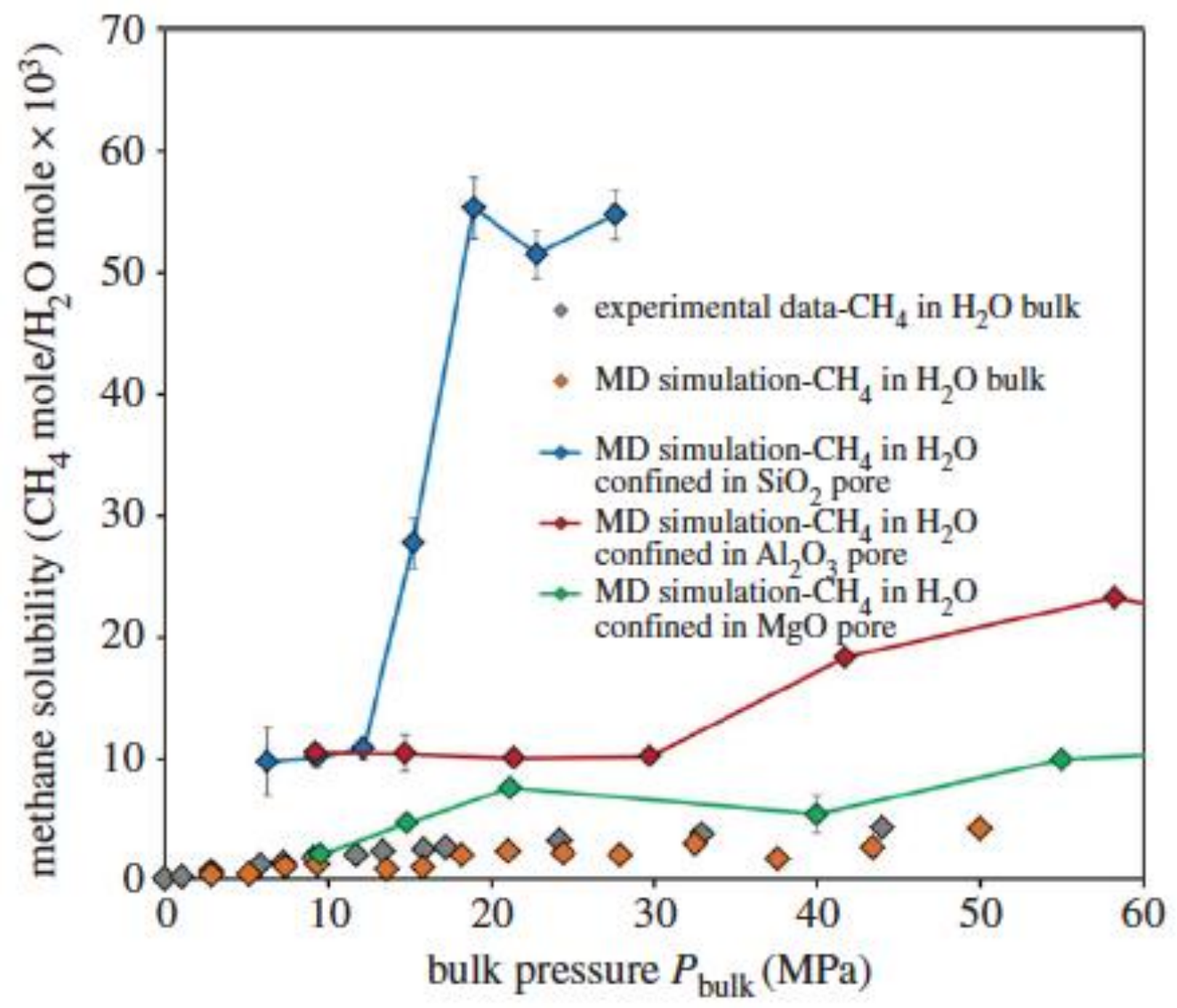

Figure 2. Methane solubility in water at ambient temperature as a function of bulk pressure. Different symbols are for different data sets. Gray and orange diamonds are experimental and simulated data for methane solubility in bulk water, respectively. Blue, red and green diamonds are simulated results for methane solubility in water confined in slit-shaped pores of $1 \mathrm{~nm}$ in width carved out of model $\mathrm{SiO}_{2}, \mathrm{Al}_{2} \mathrm{O}_{3}$ and $\mathrm{MgO}$ substrates, respectively. Reproduced from Ref. [53].

The studies discussed in relation to Figures $\mathbf{1}$ and $\mathbf{2}$ consider the situation in which water fills the entire pore volume. Such studies are expected to identify strong deviations compared to bulk properties when the pore width is so small that all the water molecules confined within the pore are strongly affected by the presence of the two solid surfaces. Under these conditions, if the mole fraction of methane present within the confined system increases with respect to that of water, one would expect a phase separation in the direction parallel to the pore surfaces. In other words, some regions would become rich in methane and depleted of water, and other regions would remain filled with water, in which methane is dissolved. Phan et al. [53] reported an example of such systems in the case of pores carved out of silica (see Figure 3). In these results it remains unclear why the interface between water-rich and methane-rich phases is irregular, as macroscopic arguments such as the 
minimization of the interfacial area would suggest that the interface should be smooth in order to minimize its length. When the pore width increases, the phase separation between methane-rich and water-rich phases is expected to occur along the direction perpendicular to the surface, so that one of the two phases could at contact with the solid substrate. For example, in the case of hydrophilic surfaces, it is expected that water adsorbs on the clay and the hydrocarbon remains near the pore center. The right panel of Figure 3 is an example of this, in the case of a pore carved out of muscovite. While for systems such as that represented on the left panel of Figure 3 it is expected that transport of methane occurs predominantly via diffusion through the adsorbed water, in systems such as that on the right panel of Figure 3 transport could be due, depending on the pressure drop, to convection. In a recent contribution, Ho et al. [55] investigated the convective transport of methane and water through narrow slit-shaped muscovite pores using non-equilibrium molecular dynamics simulations. The simulations were designed to investigate the effect of flow patterns on fluids transport. The results indicate that the Darcy's law, which describes a linear relation between flow rate and pressure drop, can be violated when the flow pattern is altered. This can happen when the pressure drop increases above a poresize dependent threshold. Such simulations illustrate the importance of the capillary force, due to the formation of water bridges across the model pores, not only on the fluid flow, but also on the pore width. When the water bridges are broken, perhaps because of fast fluid flow, the capillary force vanishes leading to significant pore
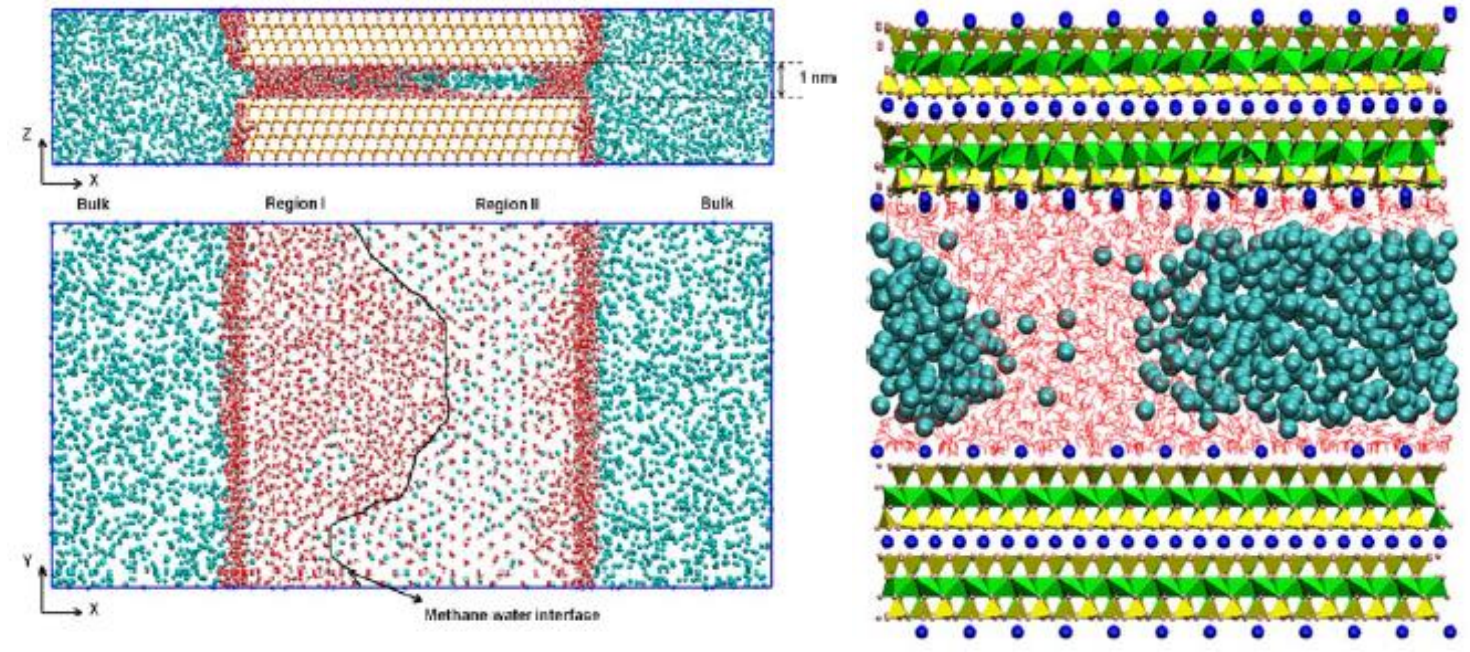

expansion.

Figure 3. Left panel: phase separation of water-rich and methane-rich phases within a narrow $1 \mathrm{~nm}$ wide slit-shaped pore carved out of $\mathrm{SiO}_{2}$. A few water molecules wet the pore surfaces also in the region where methane accumulates. Right panel: phase separation between water and methane within a slit-shaped pore carved out of muscovite. Water wets the two solid surfaces while methane occupies predominantly the pore center. A water bridge is evident between the two surfaces, but it would disappear should the water content decrease or the pore width increase. The water bridges give rise to capillary forces and affect the convective transport of methane. Reproduced from Refs. [52] and [55]. 
For simulation studies to be of relevance for the exploitation of shale gas, one should remember that in shale rocks the pores tend to be irregular, narrow, disconnected and heterogeneous [30]. Hence it is crucially important to understand the effects of defects and interconnections on the structural and transport properties of confined fluids. In addition, the fluid present within shale formations can contain water, electrolytes, naturally occurring radioactive materials, and of course hydrocarbons of different levels of maturity. In addition, the fluids used during hydraulic fracturing processes can also contain water, chemical additives, sometimes $\mathrm{CO}_{2}$ and hydrocarbons. From a fundamental point of view it is of wide interest to quantify how these different fluids behave within the heterogeneous environment of a shale formation. Generally speaking, it is accepted that hydrocarbons are contained within the kerogen inclusions dispersed within a matrix, often composed of clay, within which aqueous mixtures can often be found. However, the situation is not easily interpreted, and sometimes characterization experiments such as imbibition ones show strong dependency on the history of the rock sample [30]. This observation suggests that traces of fluids trapped within the porous network can change macroscopic observable quantities such as contact angles, capillary forces, etc.

Because of the small dimensions of the pores, and of the heterogeneous nature of the rocks, experiments are challenging, and simulations can help. However, it should again be stressed that molecular simulations can only be used to quantify phenomena that occur within very small length scales. For example, Le et al. [56,57] recently reported two sets of simulations conducted to understand how multi-component fluid mixtures behave under confinement. In these simulations the hydrocarbon (either n-octane or n-butane) was confined within a slit-shaped $\mathrm{SiO}_{2}$ pore of width $2 \mathrm{~nm}$. A range of thermodynamic conditions was explored, including temperatures ranging from subcritical to supercritical, and various densities for the confined fluids. The simulations were conducted at increasing amount of $\mathrm{CO}_{2}$. The latter compound was chosen because of its pervasive use in enhanced oil recovery applications, because in some cases it has been proposed to use super-critical $\mathrm{CO}_{2}$ to fracture shale rocks, and because geological sequestration is one of the possible strategies for the long-term management of human-made emissions. Preferential adsorption of $\mathrm{CO}_{2}$ near the $-\mathrm{OH}$ groups on the surface was observed, with the adsorbed $\mathrm{CO}_{2}$ molecules interacting simultaneously with more than one - $\mathrm{OH}$ group. This preferential adsorption acted as a 'molecular lubricant' that decreased the activation energy for the hydrocarbon diffusion within the pores, with the result that as the amount of $\mathrm{CO}_{2}$ in the system was increased, the self-diffusion coefficient of the hydrocarbons increased. Because the molecular reason for these results was the preferential adsorption of $\mathrm{CO}_{2}$ on the $-\mathrm{OH}$ groups on the surface, it is expected that confining hydrocarbon- $\mathrm{CO}_{2}$ mixtures in different rocks will yield different results, and it is likely that the presence of other fluids, e.g., water, will affect the behavior. In the case of confinement within muscovite pores, for example, MD simulations at 373K for a system containing water, $\mathrm{CO}_{2}$, and n-octane suggest that even though water covers the solid surface, $\mathrm{CO}_{2}$ distributes throughout the entire pore volume with preference 
for the pore surfaces, and n-octane is somewhat depleted near the interfacial region (see Figure 4); the main result was that the self-diffusion coefficient calculated for $\mathrm{n}$ octane decreased as the amount of $\mathrm{CO}_{2}$ present within the pore increased [58].
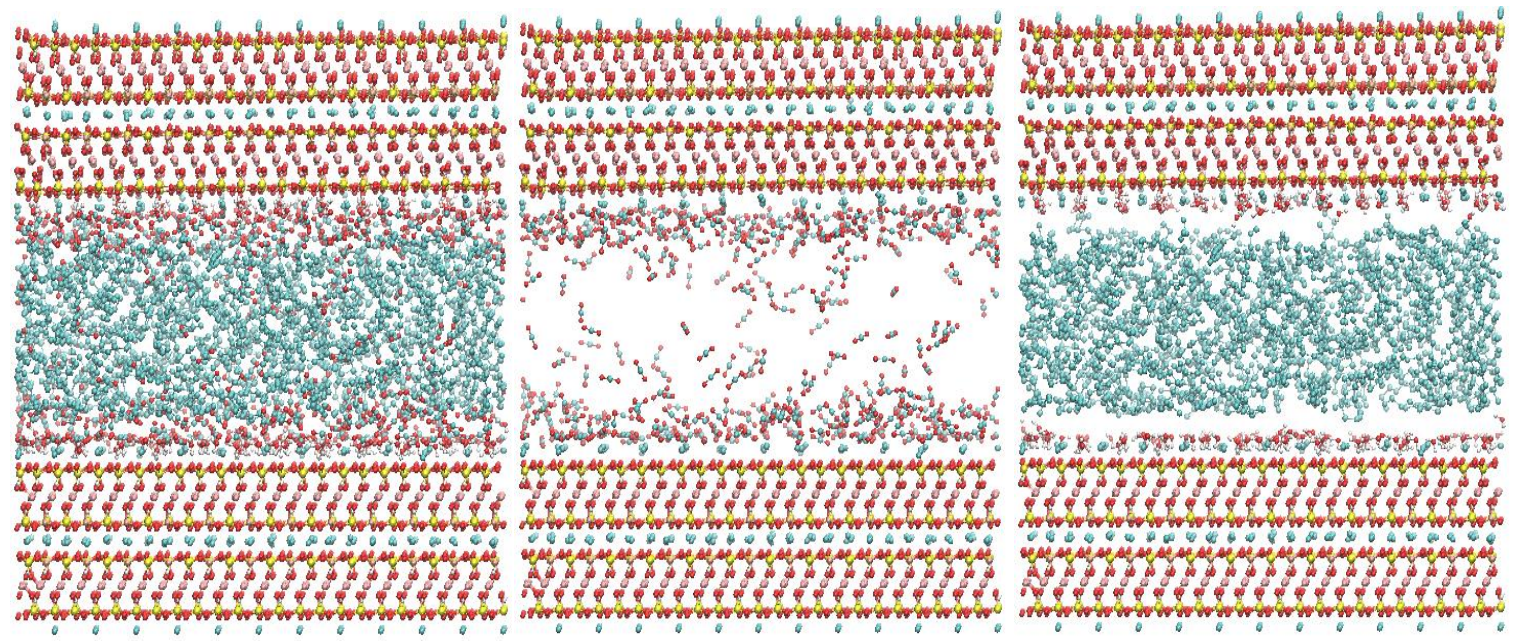

Figure 4. Representative simulation snapshots for a system containing 280 water molecules, 280 n-octane molecules, and $420 \mathrm{CO}_{2}$ molecules confined within a slit-shaped pore of width $2.8 \mathrm{~nm}$ carved out of muscovite. The simulations were conducted at $373 \mathrm{~K}$. From left to right the three panels show the complete fluid system (left), the $\mathrm{CO}_{2}$ molecules

(center), and the water and n-octane molecules (right). Cyan, red, and white spheres represent carbon, oxygen, and hydrogen atoms, respectively.

The simulation studies considered so far highlight how the properties of confined hydrocarbons, in terms of their structure, their accumulation, their preferential adsorption, and their mobility, are strongly dependent upon the nature of the confining materials, the size and shape of the pores, and the presence of other fluids, including water, sometimes even in trace amounts. Perhaps these observations help understand why experiments conducted on rock samples are sometimes difficult to reproduce, and can depend on the history of the sample.

Among chemicals that are often used by the oil and as industry, both to enhance the recovery of hydrocarbons and to remediate environmental accidents, are surfaceactive compounds, i.e., surfactants. These chemicals are used for various purposes. For example, it might be desired to adsorb surfactants at oil-water interfaces to reduce the interfacial tension; the goal might be to preferentially adsorb the surfactants on the rock surfaces to facilitate the desorption of the hydrocarbons and 'lubricate' the transport of other fluids through the porous matrix; it might also be desirable that the surfactants form reverse micelles containing hydrocarbons; etc. Such a varied range of possibilities has stimulated the thermodynamics and modeling communities for many years, not only because self-assembling systems present a number of fundamental questions that are intrinsically interesting, but also because the fundamental understanding expected from such investigations could find 
practical applications in a variety of industrial sectors, including cosmetics, nanotechnology, and drug delivery, in addition to enhanced oil recovery. As a consequence of this wide interest, much is known on surfactants behavior, adsorption, and self-assembly. However, most studies have concentrated on homogeneous systems, either in the bulk, or at contact with pristine surfaces. A number of recent discoveries suggest that perhaps the behavior of surfactants on heterogeneous surfaces differs compared to that expected based on results obtained on homogeneous substrates [59-61]. From the simulation point of view, using a coarse-grained simulation approach (dissipative particle dynamics, DPD) Suttipong et al. quantified the self-assembly of model surfactants on heterogeneous surfaces $[62,63]$. The solid substrates were either atomically flat, but with different chemical properties organized on stripes of different widths, or rough, characterized by trenches of different widths and depths. A number of unexpected features were reported. For example, when considering surfactant adsorption on patterned flat surfaces (i.e., flat surfaces with chemical heterogeneity obtained using stripes of different widths on which surfactants could adsorb surrounded by surfaces that repelled the surfactants) it was found that when the stripe is large (effectively providing a homogeneous flat surface), the surfactants yield a flat monolayer, as expected. However, the flat monolayers become hemi-cylinders, hemi-spheres, and individual surfactants as the stripe width decreases. In some cases the simulations showed evidence of cooperative effects when two narrow adsorbing stripes are not too far from each other, in which case hemi-cylindrical shells and irregular structures were observed. We are now attempting how to explain these observations based on an expression for the system free energy that accounts for the elastic deformation of the self-assembled surfactant film. For example, in Figure 5 we show representative DPD simulation snapshots to illustrate how small changes in the geometry of the solid substrate can strongly influence the morphology of the adsorbed surfactant aggregate and the amount of surfactants adsorbed.

From a different point of view, Tummala et al. [64] started to quantify how the presence of surfactants at an interface might affect the properties of interfacial water. While this level of understanding might not be directly relevant for large-scale applications in the energy sector, it can shed light on the behavior of water at interfaces, a subject that continues to be the focus of many experimental and theoretical investigations, as mentioned at the beginning of this discussion. 


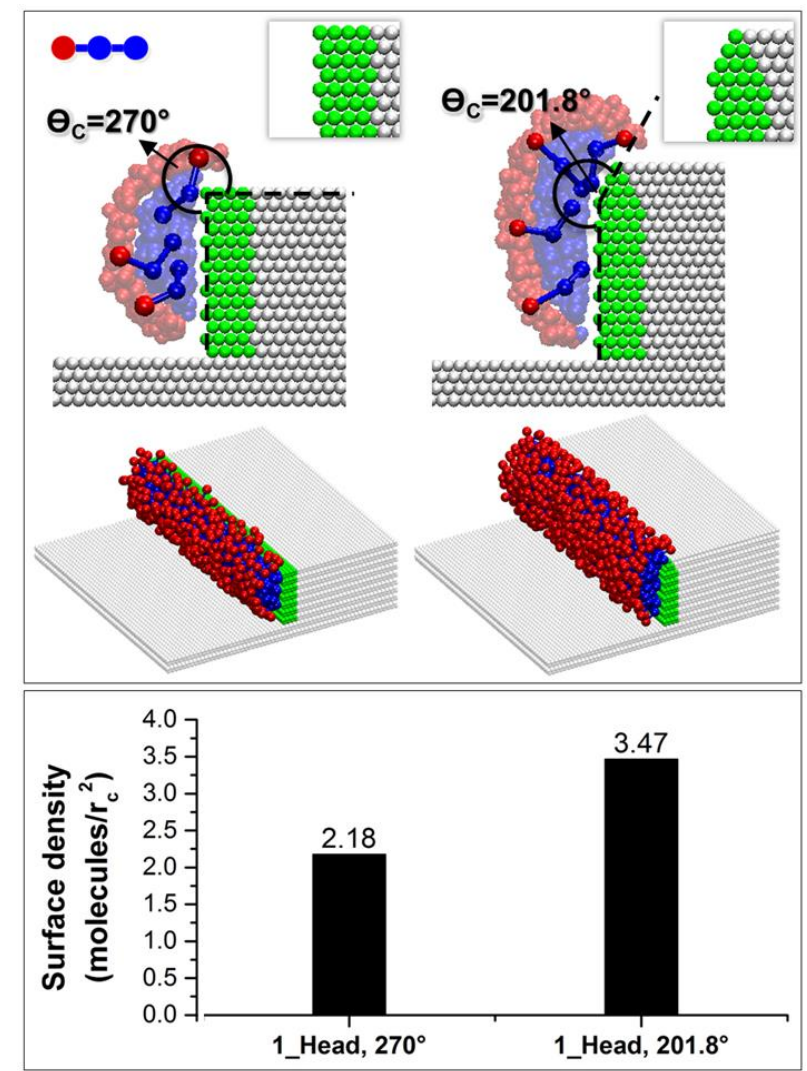

Figure 5. Top: Representative simulation snapshots for surfactant self-assembled on terraces with different pitch angles. The green region highlights the hydrophobic surface on which surfactants can adsorb. Bottom: amount of surfactant molecules adsorbed on the two terraces at constant bulk conditions. Note that the amount of surfactants adsorbed increases when the pitch angle increases, because the elastic energy due to the deformation of the self-assembled film decreases. $R_{c}$ is the unit of length in these calculations.

\section{c. Future Perspectives}

The discussion above is meant to provide a taste of how computational modeling and simulation approaches could help us to address some of the fundamental questions that remain un-answered with relation to the behavior of aqueous systems in subsurface environments. It appears that for many applications in the energy sector, including those connected with environmental preservation and remediation as well as $\mathrm{CO}_{2}$ storage, very interesting fundamental questions can have important practical applications. In my opinion, some of these fundamental questions are: (1) how do multi-component fluids partition within the complex sub-surface environment? (2) How does confinement affect and determine phase equilibria? (3) How do multicomponent fluids transport within the complex interconnected network typical of shale formations? Addressing these questions using state-of-the-art scientific approaches could become of practical use and help our society for years to come. 
Other topics that are extremely important, which stems from extremely interesting fundamental questions, which are not addressed in this brief review include, but are not limited to: hydrates, including their stability [65-67]; solubility of electrolytes in both bulk and confined water [68,69]; transport of hydrocarbons in complex sub-nm pores [70-72]; formation and dissolution of minerals [73,74]; the effect of confinement on chemical reactions at sub-surface formation conditions [75]; and many others.

When future perspectives in computational studies are discussed, it is common to highlight four typical limitations that need to be addressed, within a specific subject matter, to ensure progress. These are related to the current limitations encountered by the community, and include: (a) the accuracy of the force fields; (b) the reliability of the algorithms employed; (c) the realistic representation of the substrates considered in the calculations; and (d) the finite nature of the computational resources. For better understanding how interfacial water is related to applications in the energy sector, these four current limitations need to be addressed. In particular, in my opinion: (a) many rocks are present in the sub-surface, and it is not yet quantified how they interact with water - a combination of experiments and ab initio DFT simulations could provide data for the development of accurate fluid-rock interaction potentials, and in particular for describing defects; (b) multi-scale approaches are needed to describe fluid transport in complex porous networks and for describing simultaneous equilibria for multi-component systems throughout a variety of confining environments; (c) the resolution of experimental characterization techniques is approaching the details needed by atomistic and molecular simulations, but to secure progress a synergistic combination of experiments and computational tools is necessary; and (d) the complexity and heterogeneity of sub-surface rock formations, as well as the multitude of components simultaneously present within the environment require extremely large computational resources. The need of better understanding the fate of chemical compounds, naturally occurring radioactive matter, as well as and nuclear waste in the sub-surface should justify investments in computational capabilities.

While the discussion just summarized can be applied, with the due adjustments, to any research activities that employ multi-scale simulations as one of the key tools, the subject matter of research within the sub-surface environment has to consider a few very specific aspects. One is the scale of the applications, dictated by the depth and the physical extension of sub-surface formations. Another specific aspect to consider is the variability of the properties of the rock formations both among similar formations in different locations, and also within the same formation but at different depths. These differences can be in terms of mineral composition, fluid content, porosity, connectivity, and possibly others. Given these two aspects of uniqueness, in my opinion one should attempt to obtain general guidelines from very specific simulation studies such as those discussed here. What can we learn that can have wide applicability? While it is not clear what these generic guidelines might be at this moment, not identifying them would require ad hoc simulation studies for any specific sub-surface formation, and perhaps even for any specific site within a single 
formation. This might be possible in the future with ever more power computing capabilities, but perhaps it is an option not very attractive in the current scientific landscape.

\section{Summary}

Computational modeling and simulations have evolved tremendously over the years, because of a combination of positive impacts due to the development of reliable algorithms, accurate force fields, and computational resources. These capabilities are now implemented to address a number of fundamental questions, with applications on a variety of fields ranging from medicine, materials sciences, and also, sometimes, social sciences. The present commentary focuses on the authors' current interest in deploying computational tools to advance our fundamental understanding of relevance to applications in the energy sector. When synergistically coupled with experimental investigations, these approaches are expected to yield positive outcomes both in our ability to secure energy sources, and to limit and possibly remediate our impact on the environment. Because the studies discussed here are fundamental in nature, the results, opportunely adapted, could find applications in a variety of fields, ranging from separations, materials design and also cosmetics.

\section{Acknowledgments}

The author is grateful to all members of his research group, current and past, who carried out the work discussed here. In particular, Dr. Naga Rajesh Tummala, Dr. Dimitris Argyris, Dr. Tuan Ho, Dr. Anh Phan, Ms. Thu Le (Ph.D. candidate), and Ms. Manaswee Suttipong (Ph.D. candidate) have been instrumental for securing progress along the lines discussed here. Several undergraduate and post-graduate students at UCL have also contributed, in particular Ms. Stephanie Ogbe and Mr. Charalampos Michalakakis.

Financial support from various agencies is kindly acknowledged, in particular from the US Department of Energy, the Deep Carbon Observatory, and the European Commission (Marie Curie Career Integration Grant, and Horizon 2020 project number 640979). Financial support from Halliburton is also kindly acknowledged.

\section{References}

[1] N. Metropolis, A. W. Rosenbluth, M. N. Rosenbluth, A. H. Teller, and E. Teller, J. Chem. Phys. 21, 1087 (1953).

[2] W. W. Wood and F. R. Parker, J. Chem. Phys. 27, 720 (1957).

[3] J. A. Barker and R. O. Watts, Chem. Phys. Lett. 3, 144 (1969).

[4] A. Rahman and F. H. Stillinger, J. Chem. Phys. 55, 3336 (1971).

[5] J. K. Brennan, M. Lísal, J. D. Moore, S. Izvekov, I. V. Schweigert, and J. P. Larentzos, J. Phys. Chem. Lett. 5, 2144 (2014).

[6] N. R. Tummala, S. Mehraeen, Y.-T. Fu, C. Risko, and J.-L. Brédas, Adv. Funct. Mater. 23, 5800 (2013). 
[7] K. Wang, K. Jiang, B. Chung, T. Ouchi, P. J. Burke, D. A. Boysen, D. J. Bradwell, H. Kim, U. Muecke, and D. R. Sadoway, Nature 514, 348 (2014).

[8] J. Chmiola, G. Yushin, Y. Gogotsi, C. Portet, P. Simon, and P. L. Taberna, Science 313, 1760 (2006).

[9] X. Wang, S.-I. Choi, L. T. Roling, M. Luo, C. Ma, L. Zhang, M. Chi, J. Liu, Z. Xie, J. A. Herron, M. Mavrikakis, and Y. Xia, Nat. Commun. 6, 7594 (2015).

[10] A. K. Chakraborty and A. Weiss, Nat. Immunol. 15, 798 (2014).

[11] A. Striolo, A. Michaelides, and L. Joly, Annu. Rev. Chem, Biomol. 7, null (2016).

[12] J. H. Walther, K. Ritos, E. R. Cruz-Chu, C. M. Megaridis, and P. Koumoutsakos, Nano Lett. 13, 1910 (2013).

[13] D. Cohen-Tanugi and J. C. Grossman, Desalination 366, 59 (2015).

[14] J. M. Míguez, J. M. Garrido, F. J. Blas, H. Segura, A. Mejía, and M. M. Piñeiro, J. Phys. Chem. C 118, 24504 (2014).

[15] C. Klein, C. R. Iacovella, C. McCabe, and P. T. Cummings, Soft Matter 11, 3340 (2015).

[16] P. Morgado, G. Das, C. McCabe, and E. J. M. Filipe, J. Phys. Chem. B 119, 1623 (2015).

[17] A. Striolo, MRS Bulletin 39, 1062 (2014).

[18] A. Striolo, Adsorpt. Sci. Technol. 29, 211 (2011).

[19] N. Giovambattista, P. J. Rossky, and P. G. Debenedetti, Annu. Rev. Phys. Chem 63, 179 (2012).

[20] S. N. Jamadagni, R. Godawat, and S. Garde, Annu. Rev. Chem, Biomol. 2, 147 (2011).

[21] T. A. Ho, D. Argyris, D. V. Papavassiliou, A. Striolo, L. L. Lee, and D. R. Cole, Mol. Simul. 37, 172 (2011).

[22] T. A. Ho and A. Striolo, Mol. Simul. 40, 1190 (2014).

[23] T. A. Ho and A. Striolo, J. Chem. Phys 138, 054117 (2013).

[24] J. Ma, A. Michaelides, D. Alfè, L. Schimka, G. Kresse, and E. Wang, Phys. Rev. B 84, 033402 (2011).

[25] E. Mamontov, L. Vlcek, D. J. Wesolowski, P. T. Cummings, J. Rosenqvist, W. Wang, D. R. Cole, L. M. Anovitz, and G. Gasparovic, Phys. Rev. E 79, 051504 (2009).

[26] J. L. Achtyl, R. R. Unocic, L. Xu, Y. Cai, M. Raju, W. Zhang, R. L. Sacci, I. V. Vlassiouk, P. F. Fulvio, P. Ganesh, D. J. Wesolowski, S. Dai, A. C. T. van Duin, M. Neurock, and F. M. Geiger, Nat. Commun. 6, 6539 (2015).

[27] D. Argyris, A. Phan, A. Striolo, and P. D. Ashby, J. Phys. Chem. C 117, 10433 (2013).

[28] D. Argyris, P. D. Ashby, and A. Striolo, ACS Nano 5, 2215 (2011).

[29] A. Yethiraj and A. Striolo, J. Phys. Chem. Lett. 4, 687 (2013).

[30] V. Marry and B. Rotenberg, in Developments in Clay Science, edited by Christophe Tournassat, Carl I. Steefel, Ian C. Bourg, and Fappa Bergaya (Elsevier, 2015), Vol. 6 pp. 399-417.

[31] J. A. Greathouse and R. T. Cygan, Environ. Sci. Technol. 40, 3865 (2006).

[32] V. Marry, B. Rotenberg, and P. Turq, Phys. Chem. Chem. Phys. 10, 4802 (2008).

[33] C. Tournassat, Y. Chapron, P. Leroy, M. Bizi, and F. Boulahya, J. Colloid Interface Sci. 339, 533 (2009).

[34] I. C. Bourg and G. Sposito, J. Colloid Interface Sci. 360, 701 (2011). 
[35] M. Makaremi, K. D. Jordan, G. D. Guthrie, and E. M. Myshakin, J. Phys. Chem. C 119, 15112 (2015).

[36] A. Z. Panagiotopoulos, Mol. Phys. 62, 701 (1987).

[37] A. Botan, B. Rotenberg, V. Marry, P. Turq, and B. Noetinger, J. Phys. Chem. C 114, 14962 (2010).

[38] J. O. Titiloye and N. T. Skipper, J. Colloid Interface Sci. 282, 422 (2005).

[39] X.-D. Liu and X.-C. Lu, Angew. Chem., Int. Ed. 45, 6300 (2006).

[40] R. K. Kalluri, M. M. Biener, M. E. Suss, M. D. Merrill, M. Stadermann, J. G. Santiago, T. F. Baumann, J. Biener, and A. Striolo, Phys. Chem. Chem. Phys. 15, 2309 (2013).

[41] T. Le, A. Striolo, and D. R. Cole, Chem. Eng. Sci. 121, 292 (2015).

[42] M. Jardat, J.-F. Dufreche, V. Marry, B. Rotenberg, and P. Turq, Phys. Chem. Chem. Phys. 11, 2023 (2009).

[43] D. Konatham, J. Yu, T. A. Ho, and A. Striolo, Langmuir 29, 11884 (2013).

[44] B. R. Bickmore, K. M. Rosso, K. L. Nagy, R. T. Cygan, and C. J. Tadanier, Clays Clay Miner. 51, 359 (2003).

[45] S. V. Churakov, J. Phys. Chem. B 110, 4135 (2006).

[46] A. Kremleva, S. Krüger, and N. Rösch, Geochim. Cosmochim. Acta. 75, 706 (2011).

[47] X. Liu, X. Lu, M. Sprik, J. Cheng, E. J. Meijer, and R. Wang, Geochim. Cosmochim. Acta. 117, 180 (2013).

[48] T. R. Zeitler, J. A. Greathouse, J. D. Gale, and R. T. Cygan, J. Phys. Chem. C 118, 7946 (2014).

[49] R. T. Cygan, J.-J. Liang, and A. G. Kalinichev, J. Phys. Chem. B 108, 1255 (2004).

[50] D. Argyris, D. R. Cole, and A. Striolo, ACS Nano 4, 2035 (2010).

[51] T. A. Ho, D. Argyris, D. R. Cole, and A. Striolo, Langmuir 28, 1256 (2012).

[52] A. Phan, D. R. Cole, and A. Striolo, J. Phys. Chem. C 118, 4860 (2014).

[53] A. Phan, D. R. Cole, and A. Striolo, Phil. Trans. R. Soc. A 374, 20150019 (2016).

[54] P. Giesting, S. Guggenheim, A. F. Koster van Groos, and A. Busch, Int. J. Greenh. Gas Control 8, 73 (2012).

[55] T. A. Ho and A. Striolo, AIChE J. 61, 2993 (2015).

[56] T. Le, A. Striolo, and D. R. Cole, J. Phys. Chem. C 119, 15274 (2015).

[57] T. Le, S. Ogbe, A. Striolo, and D. R. Cole, Mol. Simul., (2016) in press.

[58] C. Michalakakis, MEng thesis, University College London, 2014.

[59] H. C. Schniepp, H. C. Shum, D. A. Saville, and I. A. Aksay, J. Phys. Chem. C 112, 14902 (2008).

[60] S. Wu, L. Shi, L. B. Garfield, R. F. Tabor, A. Striolo, and B. P. Grady, Langmuir 27, 6091 (2011).

[61] N. R. Tummala, B. P. Grady, and A. Striolo, Phys. Chem. Chem. Phys. 12, 13137 (2010).

[62] M. Suttipong, B. P. Grady, and A. Striolo, Phys. Chem. Chem. Phys. 16, 16388 (2014).

[63] M. Suttipong, B. P. Grady, and A. Striolo, J. Phys. Chem. B 119, 5467 (2015).

[64] N. R. Tummala, S. Liu, D. Argyris, and A. Striolo, Langmuir 31, 2084 (2015).

[65] V. K. Michalis, J. Costandy, I. N. Tsimpanogiannis, A. K. Stubos, and I. G. Economou, J. Chem. Phys. 142, 044501 (2015). 
[66] L. Cai, B. A. Pethica, P. G. Debenedetti, and S. Sundaresan, Chem. Eng. Sci. 141, 125 (2016).

[67] S. Sarupria and P. G. Debenedetti, J. Phys. Chem. Lett. 3, 2942 (2012).

[68] Z. Mester and A. Z. Panagiotopoulos, J. Chem. Phys. 142, 044507 (2015).

[69] W. R. Smith, F. Moučka, and I. Nezbeda, Fluid Phase Equilibria 407, 76 (2016).

[70] S. Müllegger, M. Rashidi, M. Fattinger, and R. Koch, J. Phys. Chem. C 116, 22587 (2012).

[71] K. Falk, B. Coasne, R. Pellenq, F.-J. Ulm, and L. Bocquet, Nat. Commun. 6, 6949 (2015).

[72] K. Falk, R. Pellenq, F. J. Ulm, and B. Coasne, Energy Fuels 29, 7889 (2015).

[73] A. Putnis, Science 343, 1441 (2014).

[74] N. Laanait, E. B. R. Callagon, Z. Zhang, N. C. Sturchio, S. S. Lee, and P. Fenter, Science 349, 1330 (2015).

[75] C. Heath Turner, J. K. Brennan, M. Lísal, W. R. Smith, J. Karl Johnson, and K. E. Gubbins, Mol. Simul. 34, 119 (2008). 